\title{
Energy Representation Tool for Air Conditioning that Enhance Energy Savings and Improve Energy Literacy on Users
}

\author{
Roberto García-Manzano ${ }^{1}$, Alfonso P. Ramallo-González ${ }^{1}$, Ramón Sanchez-Iborra ${ }^{1}$ \\ ${ }^{1}$ Universidad de Murcia, Murcia, Spain
}

\begin{abstract}
Augmented reality can be considered as a nascent field for building simulation of variables that are not visible for the naked eye. Luckily, the proliferation of smart-phones and the developments on Augmented Reality (AR) are opening new avenues for the better information transfer to these users. For energy and conditioning, AR can offer tools for improving energy literacy at home. This work shows the evaluation of four different $\mathrm{AR}$ methods for the representation of energy consumption by $\mathrm{A} / \mathrm{C}$ machines. These methods use simple analogies instead of letters or numbers. The systems have been tested with 25 participants with heterogeneous demographics with the SUS survey, and it has been seen that there is a large will to use these systems among the participants in all demographic groups. It has been proven that depending on the demographic group, participants preferred specific representations which keep a high level of correlation with age. There is therefore a high customization potential for this type of services. This makes the technology highly interesting as its cost is virtually null in users with a smartphone and an $\mathrm{A} / \mathrm{C}$ machine connected to the Internet (as the latest models available in the market).
\end{abstract}

\section{Introduction}

Augmented reality can be defined as the superposition of virtual elements over a vision of the reality, so that it adds additional information to that reality. The birth of augmented reality is linked to the virtual reality from its inception and will not be until later, when the augmented reality is sufficiently perfected to separate the two branches. In this work, augmented reality has been considered as a vehicle to improve energy literacy since the reduction of energy consumption in buildings is essential to meet the emission reduction objectives, which has been marked, among other actions, as essential by the European Union (Energy Efficiency Directive Recast, 2011). It has been established that buildings are responsible for up to $40 \%$ of emissions in developed countries (Pérez Lombard, Ortiz y Pout, 2008).

People are not aware of energy waste, partly because of a lack of energy literacy (Jervey, 2012). There are initiatives such as the FIESTA project, financed by the Intelligent Energy-Europe Program, whose main purpose is to promote new daily consumption habits that are more energy efficient, especially in relation to the use of heating and air conditioning, given that the spending on air conditioning represents $66 \%$ of the total use of energy in the home (Fiesta-audit.eu, 2018). To achieve this change of habits that leads to a better energy consumption, it is necessary to put the user in perspective, providing the necessary information in a simple and direct way.

The user cannot manage what he cannot measure. Making visible the invisible attracts people's attention (Gardner and Stern, 2002), cited by Pahl (2016), that is why in this work an application for mobile phones and tablets called "Panoptic" has been developed. It makes use of twodimensional codes (QR (Quick Response) Codes) and augmented reality with the aim of showing energy consumption in real time of air-conditioning consoles. Four different visualization models have been designed and implemented, and they have been evaluated by 25 users. The results of the tests have been collected through a questionnaire that includes, in addition to the demographic profile of the person, questions about their attitude towards the use of energy, a standardized test on usability of the system (SUS - System Usability Scale) and various questions about the visualization models.

The first study to be highlighted is that in (Pelliccia et al., 2015). This study proposed a power visualization system for industrial machinery. In the study (Rashed-Ali et al., 2014), researchers focused on passive energy efficiency. An application was developed that allowed placing a house on a plot of land and changing the parameters in terms of orientation of the house, size, proportions or shade, in addition, the user can select the time of day or the season of the year.

The rest of this paper is organized as follows. Section II presents the methodology followed in our experiments. The attained results are showed and discussed in Section III. The paper ends in Section IV highlighting the most important findings.

\section{Methodology}

This work includes a field work experimentation to evaluate a series of methods of interpretation of energy used by an air conditioning unit using augmented reality. This has been developed as a mobile application with several choices of interpretation, a questionnaire to evaluate the impression of the participants and the usability of the system has been done with the subsequent statistical analysis of the data. 


\section{Mobile application}

The functionality is based on the reading of a QR code that has been placed in each one of the devices to be monitored, this code includes a unique identifier that will be used to consult, through an API, your current consumption level. The panoptic application has been developed using Unity3D, a free engine for crossplatform applications. The reason for using this technology is to facilitate integration in a variety of applications. This tool can be used to create applications (primarily games) both in $3 \mathrm{D}$ and $2 \mathrm{D}$, cross-platform, using a language of scripting in C\# or Javascript.

The Vuforia project, allowed to include Virtual Reality within a project created with Unity. Vuforia is an augmented reality library for mobile SDK. It uses computer vision to recognize and make tracking of flat images and simple objects in 3D in real time. It allows to position and orient virtual objects, such as 3D models, in relation to the real world in such a way that the user can navigate through the scene keeping the perspective of virtual object within the real world, leaving it completely integrated into the scene. The library of open source ZXing that allows to read QR codes was also integrated.
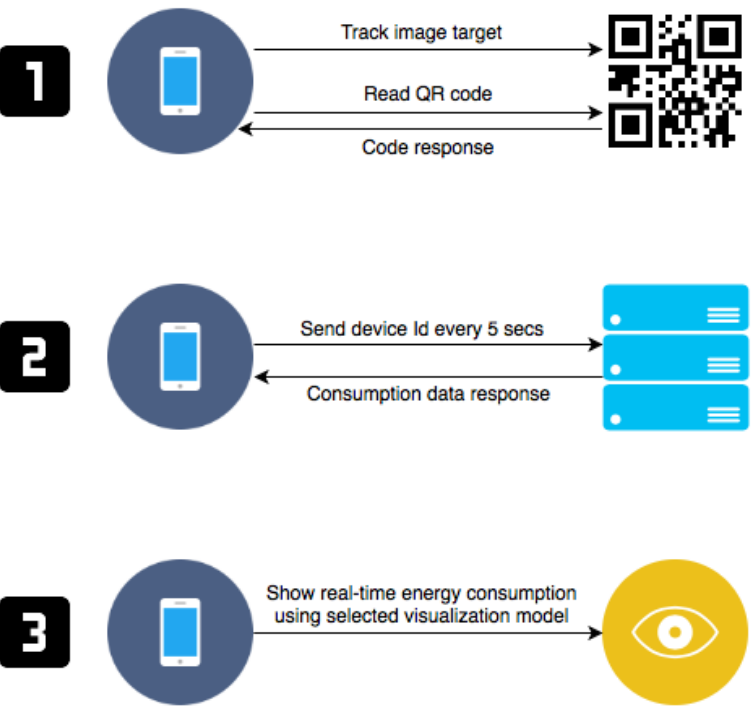

Figure 1: Schematic view of the working mechanism of PANOPTIC.

The augmented reality system begins when the user focuses the camera of the phone or Tablet in the QR code that contains the identifier associated with the device, at this time the application communicates with the given servers to download the associated information and begins a polling process every 5 seconds by which the consumption information will be refreshing.

To have real numbers, data from an air conditioning machine, was used to determine the level of consumption with respect to room temperature and the temperature of the thermostat. We obtained this from a building model which details can been seen in (Ramallo-González et al. 2019). From these data, it is possible to calculate the level of current consumption with equation (1):
$\mathrm{C}=2667+48, *(\mathrm{Ts}-\mathrm{Tt})$

Being:

- Ts: current room temperature.

- Tt: temperature set on the thermostat.

The constants were obtained through a simulation of air conditioning machines of the University of Murcia using the EnergyPlus7 software.

\section{Representation models}

As mentioned before, in the related literature little was found on the application of augmented reality in the visualization of energy systems or as a tool for the improvement of building simulation results visualisation.

As a first goal, you want to design an attractive display model to show the consumption in real time from a particular device. To ensure that interpretations are being studied with a large target audience, the system should be simple and direct. It was considered (and it will be evaluated with the questionnaire) that a good approach would be to support the information in metaphors rather than actual numbers so that it can be understood quickly regardless of prior knowledge of the user.

The development of this study has been designed and implemented a mobile application of 3D augmented reality called panoptic (/ pan'pptik /) meaning, according to the Oxford Dictionary: "showing or seeing the whole at one view".

The four different models of representations are as it follows:

Particles based model

For the first model the system of particles of Unity has been used to show the use of the device in real time. Figure 1 shows the model showing a low consumption level.

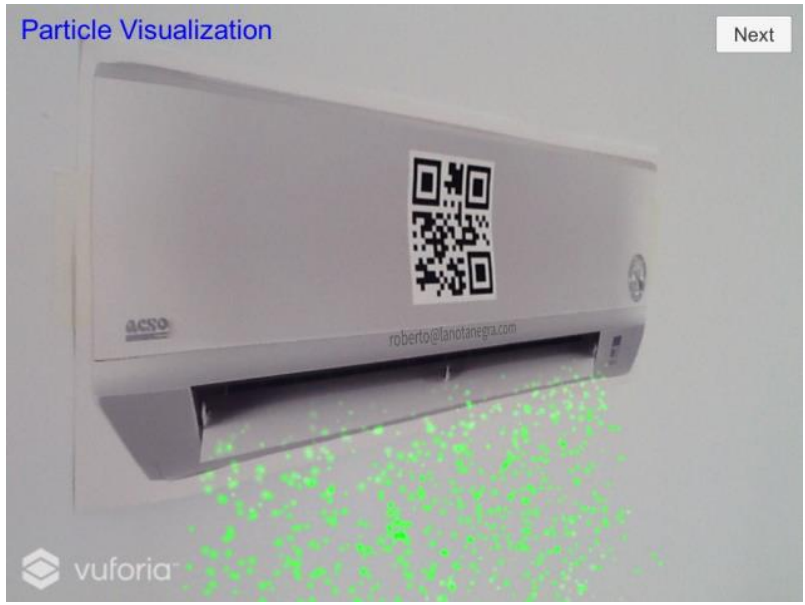

Figure 2: Particles-based visualisation method.

Different levels of consumption are represented as:

- Low level: green and with a speed of movement of the particles of 2 .

- Medium level: yellow and with a speed of simulation of 3 . Increasing the speed also increases the ratio of particles that are currently displayed in the visualisation. 
- High level: red and with a speed of simulation value 4. As in the medium level, faster consumption, greater number of particles.

Also the values of "velocity over time" and "colour over time" have been adjusted so that the movement of the particles is as natural as possible. The effect is very similar to the soft snow fall at low speeds and something more akin to a storm when the speed is determined by the level of high consumption. In addition, by adjusting the values of opacity, size, colour, and speed of the particle is possible to control their behaviour.

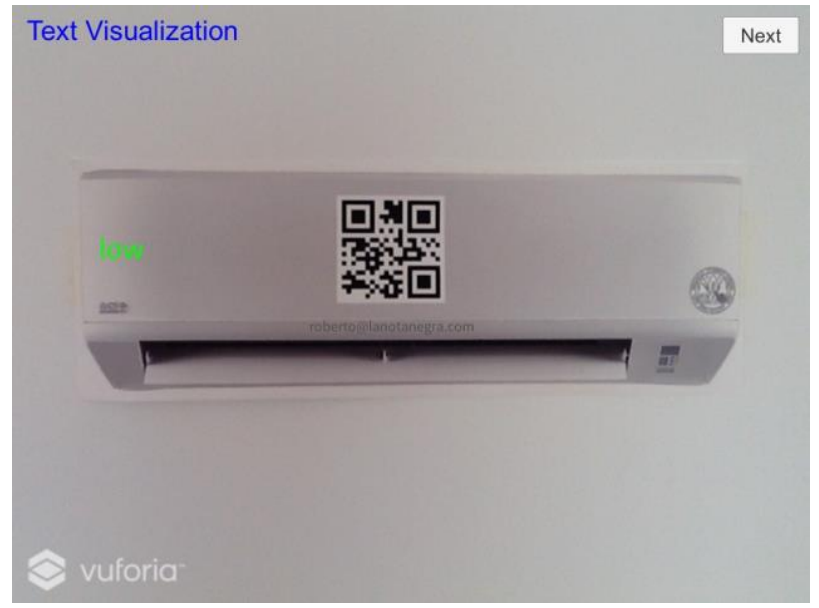

Figure 3: Text-based visualisation method.

\section{Text-based model}

The text-based model is a simple model of display which only shows a text in 3D stack on the device label. The label indicates what the current consumption is:

-Low Level: green label showing the word "low".

-Medium Level: yellow label showing the word "moderate".

-High Level: label in red showing the word "high" accompanied by a recommendation for the user to adjust the level of the thermostat.

The model for a low consumption level is shown in Figure 2.

\section{Surface based model}

In the Surface-based model, the entire surface of the device which is being measured changes colour and opacity depending on the level of consumption. With a level of average consumption model is shown in Figure 3. Depending on the level of consumption behaviour is as follows:

-Low Level: the surface of the device is green, with a level of opacity of 0.5 .

-Medium Level: the surface of the device is yellow, with a level of opacity of 0.75 .

-High Level: the surface of the device is red, with a level of opacity of 1 , i.e., at this level the device already it will not be visible, but a completely solid representation of it in red.

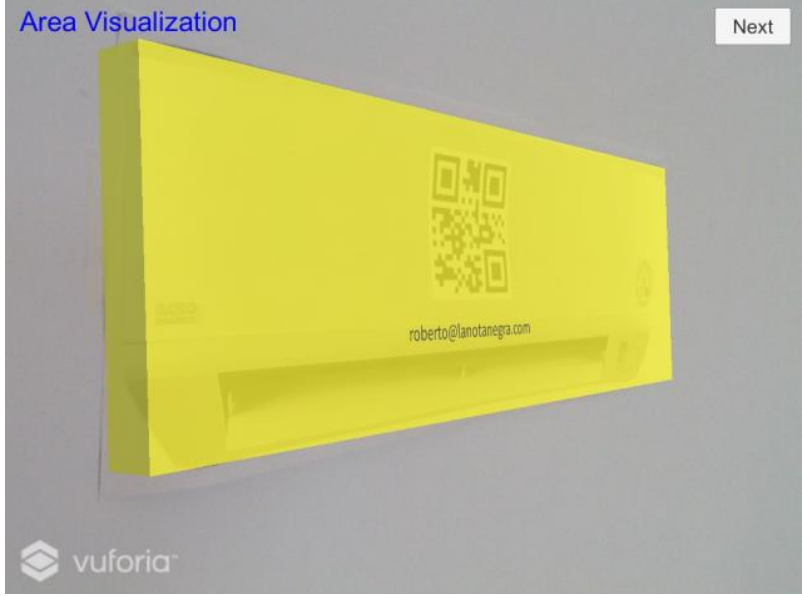

Figure 4: Surface-based visualisation method.

\section{Sound-based model}

This is the only metaphor that is not visual and is due to two reasons: on the one hand, you want to see the effect that produces an audible warning regarding levels of perception of consumption; and on the other hand it is also necessary to make the application to those persons with certain visual disabilities accessible. This model depending on consumption behaviour is as follows:

- Low level: the system emits a short beep.

- Middle level: System emits three short beeps.

- High level: playing on loop the middle level until the situation is corrected, or until the user stops focusing on the device.

As shown in the section of the analysis of the results, sound-based model shows a level of understanding of the very low current consumption, but gives the greater sense of urgency prints the users to correct a situation of high consumption.

\section{Experimental design}

Once developed the augmented reality system, it has undergone evaluation by a group of users.

To evaluate the performance of different models of visualization, usability and the viability of the project, a questionnaire has been developed which has been performed using a cohort of 25 users (14 men and 11 women), aged between 21 and 78 years, with heterogeneous income and levels of education. Users were recruited through invitation. And the test was done into a test room without incentives of any kind for participating in the test.

Users evaluated the usability of the system and other aspects according to the standardized test SUS. The questionnaire consists of four parts:

- Demographic profile: includes basic questions on age, sex, level of education, as well as data on monthly earnings.

- Attitude towards the use of energy: is made up of statements assessing the usage and customs of the user regarding the use of energy. In this section, questions have been included to assess the social aspect of the behaviour of the participants. 
- System usability test: is assessed using a standardized test widely used in the industry as it is the System Usability Scale or its. It consists on a quick questionnaire that allows you to measure the usability of a system, even if the number of participants is not too high. Designed by Brooke (1986) and used as a standard in the industry, it has been referenced in more than 1300 articles and publications (Usability.gov, 2013).

- Evaluation of displays: includes statements about the impact of each of the displays, the levels of urgency to fix a level of high consumption and comparisons between them.

The test was performed in a test room that consists of a real room of $35 \mathrm{~m}^{2}$ located in the city of Murcia. The test was conducted during the months of July and August.

In the test room is available a poster with a replica of the conditioned air in the different faculties of the University of Murcia. The machine has a QR code with an identifier that will be later used to collect consumption. The reason for this design is to extend this functionality to more than 400 air conditioning machines available in the University of Murcia.

In terms of communication of the components of the experiment, orders are sent to an API (Application Programming Interface) testing which changes in the level of consumption have occurred, being automatically reflected in the application. In addition, to avoid losing the environmental component, also an air conditioning machine is present in the room, so real temperature perceptions are perceiving by the participant. The interviews are conducted on an individual basis, with the room at an ambient temperature of $23^{\circ} \mathrm{C}$.

The session with the participant starts with the user by filling in the first two sections of the questionnaire: demographic profile and attitudes to the use of energy. Once users are ready to begin, they are given a tablet with the application installed and opened. The only instructions given to users is that to start the test (not guided) should focus on the poster containing the replica of the air-conditioning machine.

The first display that the user faces is the model based on particles, showing each of the different levels of consumption. Then the user will use models based on text, surface and sound. When the user has finished the test, they can fill the usability test and evaluation of each of the display models.

\section{System usability scale (SUS)}

This test provides a reliable tool to measure usability. It consists of a ten-item questionnaire with five options of response in a Likert scale; from "completely agree" to "totally in disagreement".

As already said in the presentation of the experiment, the test has become an industry standard. Its benefits are:

- It is a scale that is easy to use for the participants.

- It can be used with a small population, obtaining reliable results.
- It is verified, i.e., it can determine effectively between systems usable and not usable. The score of each of the answers is converted to a new number: added together and then multiplied by 2.5 to convert the original scale (0-40) to the end (0-100). Although the scale reaches 100, they are not percentages.

Based on studies (Sauro, 2011), a score above 68 would be considered to be above the average, and any score below 68 would be below the average.

Bangor, Kortum and Miller (2011) in his study "Determining individual what your scores mean: Adding an adjective rating scale", established a scale which accounted for scores and their corresponding adjectives or names. They also established the score by which separate the usability of "good" systems of the "not so good". This cohort is located in the score of 71.4. Any score above 71.4 is considered a system whose usability is above the average, and any score below 71.4 corresponds to a system whose usability is below the average

\section{Results}

To evaluate the results of the experimentation, a series of statistical evaluations were done and are explained in this section.

\section{Desirability}

The analysis of the results begins with the intention of use of users depending on their demographic profile and other questions. Figure 4 shows that women who underwent the assessment have an increased willingness to use the system than men, although the Mann-Whitney test confirms there is no statistical significance with a p-value $=0.581$.

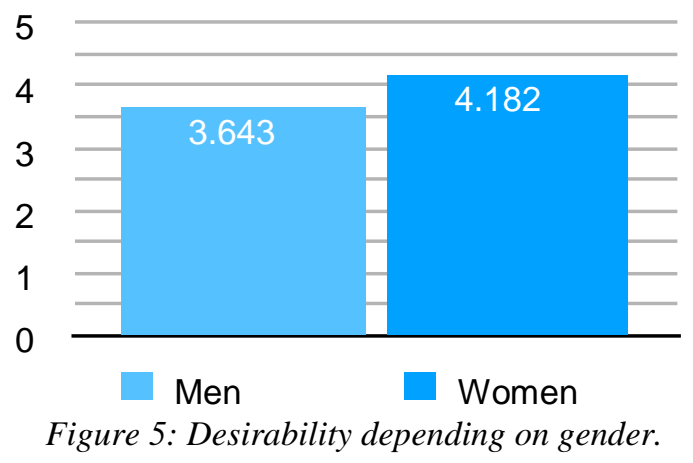

Figure 5 shows as, in general, there is a high willingness to independently use an augmented reality system despite the monthly net income of the family. The willingness to use is inversely proportional to the level of the family income, i.e. those families having less income are which require more information to adjust their consumption. It is true that there is a decline in the willingness to use in the range from 1,500 to $€ 1,999$ monthly income, this may be due to a low of subjects that meet this criterion since the sample is not very large. Making the Kruskall-Wallis test you get a p-value of 0.662 indicating that a relationship between income level and willingness to use in not significant. 


\section{5}
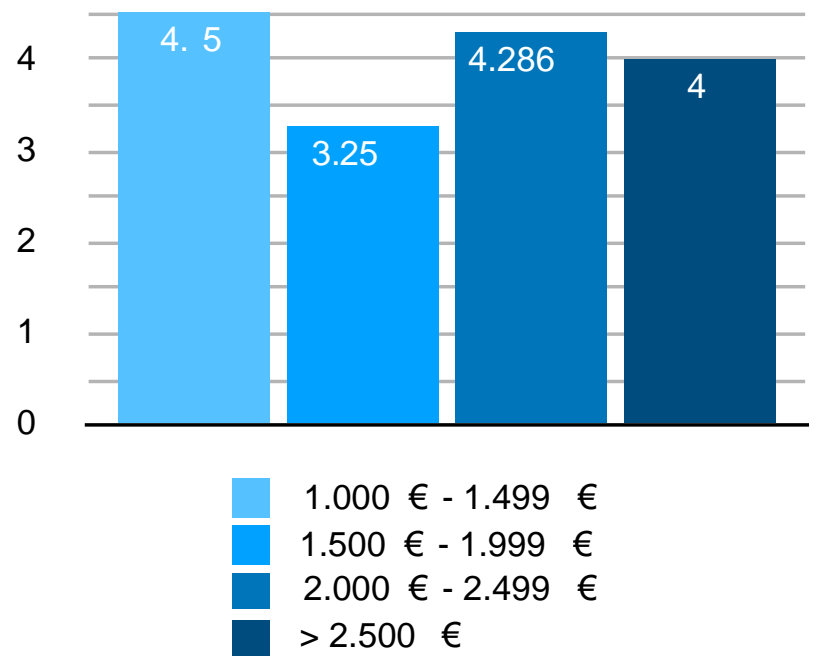

Figure 6: Desirability depending on income.

The willingness of use the tool is also very high regardless of the academic level. (Figure 6). Although this time the Kruskall-Wallis test confirms that a correlation exists between the level of studies and willingness to use the system $(p$-value $=0.0215)$. At the same time, at first glance there is no significant difference in the willingness to use depending on who pays the bills, the MannWhitney test yields a p-value of 0.145 by what we can confirm that this is so.

Figure 7 shows a graph comparing willingness to use with different questions of the section "attitude towards the use of energy", it makes reference to "save energy is something I do frequently", ACT_K is "I would consider better person if I wore less energy" and ACT_N is "If you have more information about my energy consumption would make a more responsible use".

It can be seen that those who said that they saved energy frequently with one value greater or equal to 3 on 5 present a great willingness to use the system, those who claim a 2 are not interested in using the system, the Kruskall-Wallis test confirms that both claims are related to a $95 \%$ confidence level ( $p$-value $=0.002$ ). In the same way as in the previous question, it seems that users who indicated that feel better saving energy giving a score would be equal to or greater than 3 out of 5 show great willingness to use the system, but using the above test yields a p-value of 0.135 by what this assumption should be discarded.

For the statement that indicates that the user would make a better use of energy if he has more information, also the willingness to use the system is correlated. For higher scores or equal to 4 out of 5 the test confirms this with a p-value of 0.006 . Curiously, none of the respondents marked with 5 out of 5 to the assertion "saving energy is something I do frequently". From this first part of the analysis, it can be inferred that the will to use the system by users, in general terms, is quite high.

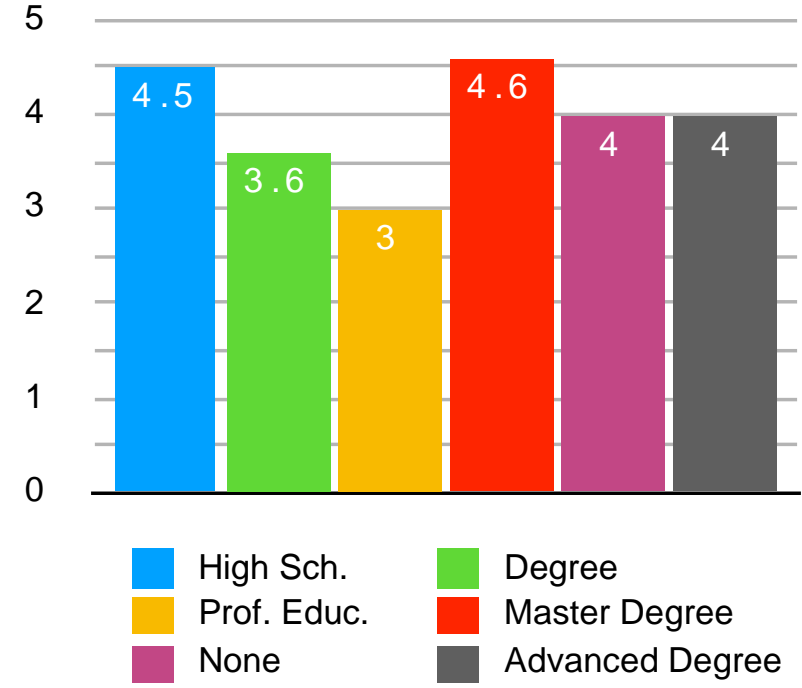

Figure 7: Desirability depending on academic level.

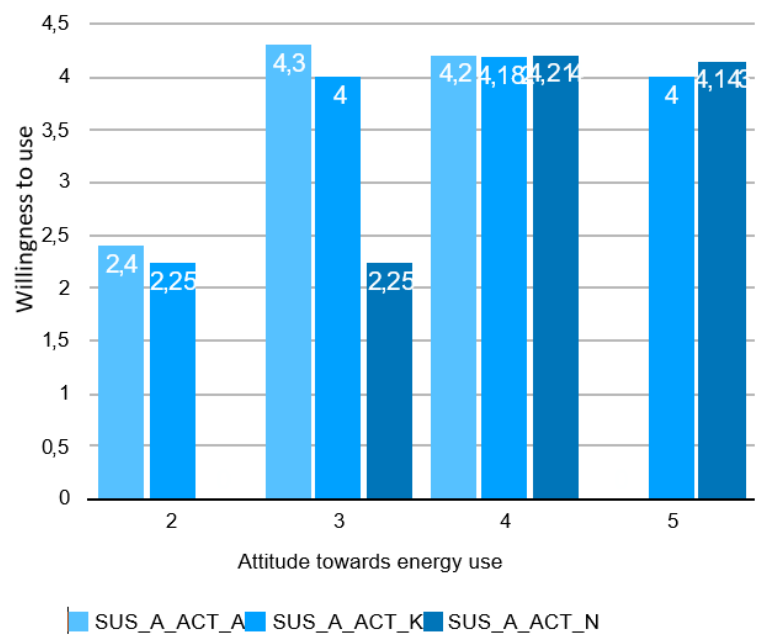

Figure 8: Desirability depending on attitudes towards energy.

\section{Usability Test}

The evaluated system has an average of 89.6 rating so it can be considered "excellent" on the proposed scale, and very close to the category "best imaginable". Figure 8 shows the distribution of scores. The majority of scores in the test have fallen into the bucket, 90-99, which demonstrates that the designed augmented reality system excels in usability with a fairly high note.

\section{Results depending on the visualisation model}

This section will analyse the results of the different display models proposed and evaluated by users. In Figure 9 the results of the first item of the questionnaire related to models are shown. The statement was related to the speed with which the level of consumption was understood when it was high. The text-based model is above the rest followed by the model of particles and surface. Sound-based model is the worst in this aspect. 


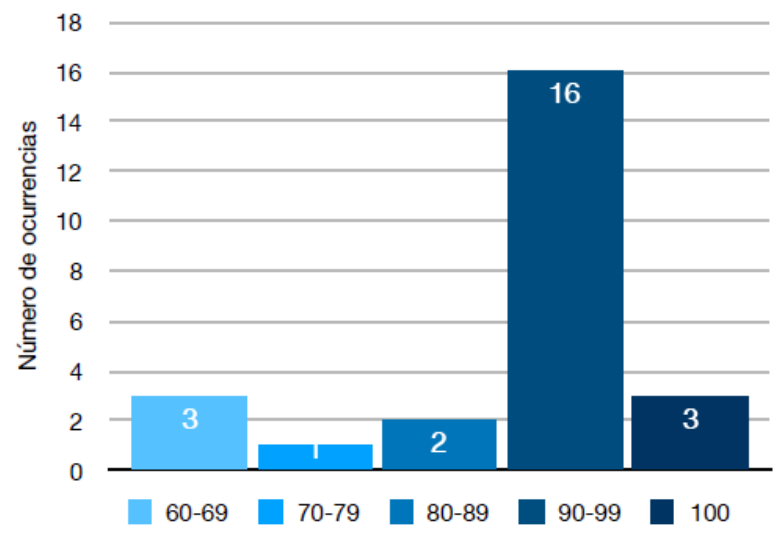

Figure 9: Scores on the SUS.

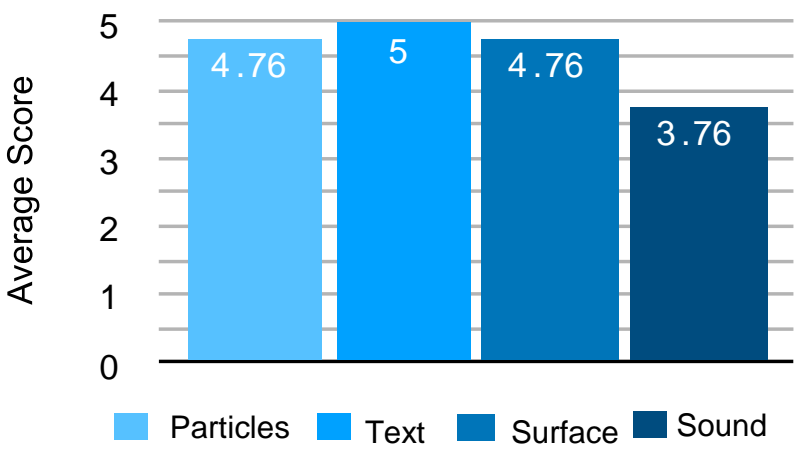

Figure 10: Answers to the statement: I have understood fast the consumption when observing a high level.

In Figure 10 the results of the second item in the test evaluation of the models is shown. In this case, the question is related to the satisfaction that it is felt when the consumption is shown to be low. A rather moderate satisfaction is seen when showing low consumption level in general. The highest score was the surface model; it might be because in this model the display occupies a larger percentage of the screen with green colour. As shown in Figure 10 with none of the models of display users have felt a special urgency to act to correct a moderate energy consumption, once again, the model which slightly stands out above the rest is the surfacebased model. In Figure 10 is observed clearly as the model is the one that encourages the user to correct a high consumption.

In Figure $11 \mathrm{t}$ is shown a breakdown of the visualization method preferred for the age range. Continuing with the affirmation of the previous paragraph, it comes with no surprise that users that fit in the lower age ranges do prefer more Visual models and those who are in the upper age ranges, prefer more direct models as based on text.

As a conclusion to the sections of results, it is correct to assert that augmented reality system have achieved a score well above the average in terms of usability despite display model chosen, but if any of them outstands by the majority of users this has been the model based on particles. It is also true that the model that most urgently moves users to correct a situation of high energy consumption has been the model based on sounds, so perhaps the model idea is one that combines an attractive

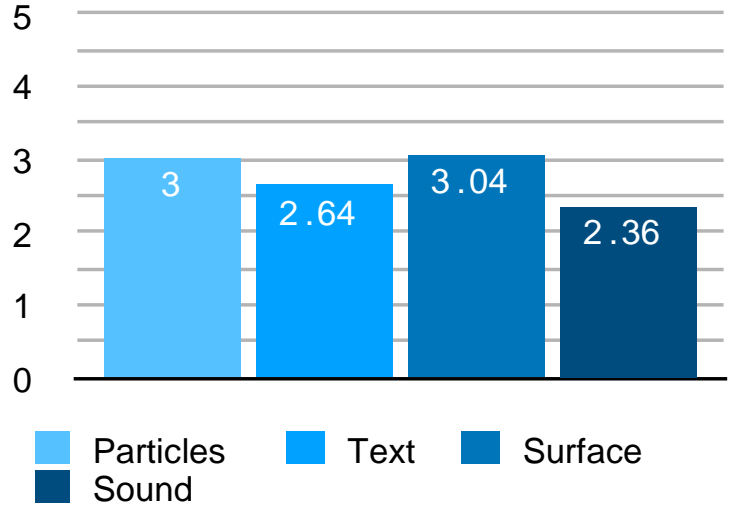

Figure 11: Answers to the statement: I have felt satisfied when I see a low level.

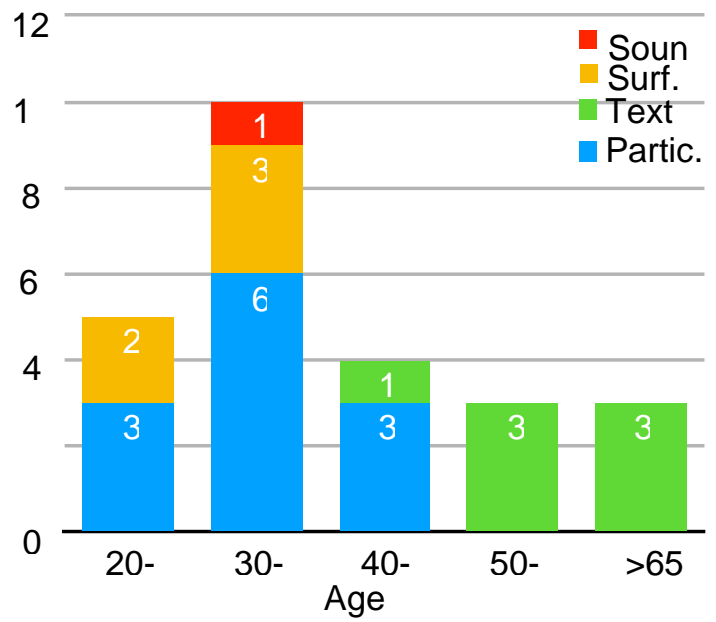

Figure 12: Favourite model depending on age group.

display as the model based on particles with a more "annoying" model as the sounds to correct high levels of consumption.

\section{Conclusion}

In the literature consulted about the topic nothing similar to this study was found, so it is likely that this paper opens a new avenue of research. It was found that joining augmented reality with a lack on energy literacy allows the user to understand in real time and on demand, the energy consumption of certain devices. There are also other studies that aim to promote energy literacy of users and efficiency of devices emerging in the industry, so the interest is justified.

With the tests carried out here it was found that, among users who had the test, there is a great willingness to use this type of technology to visualize and thus controlling energy use in the home. It has been shown that, among the surveyed population, both men and women have a very high willingness to use the augmented reality system, moreover, the results confirm that an inverse correlation exists between the monthly income of the family unit and the willingness to use.

Statistical tests have also confirmed that the willingness to use the system is directly linked to the educational level of the users, the highest interests are concentrated at the higher educational levels. In addition, there is no 
difference at will when determining who is responsible for paying the bills. The participants who claimed that they were saving energy were more willing to use the augmented reality system. The same applies to persons who claim that they feel better when saving energy. Also a high intention to use the system is seen in those who scored above 4 out of 5 to the statement which indicates that the user would make better use of energy if their have more information about their consumption.

According to the results obtained in the analysis of the willingness to use the system, it is possible to conclude that, in general terms, it is quite high, and very transversal. In terms of the evaluation of the usability of the developed system, even considering that it is still at a very early stage, it has shown a usability far above expectations, obtaining a score of 89.6 points on the test and an "excellent" rating, very close to the "best imaginable".

Within the display models proposed for evaluation, users have chosen the model based on particles as the one that more impact generates on them. They were not especially motivated with specific proposed models to correct a high consumption level, but if they claim that they felt a special urgency to correct a high consumption level it was when the sound-based model is presented to them. A priori, it seems that models based on particles with sound alerts when consumption is high, seems to be the best option.

In conclusion and observing all the results, it can be said that it is possible to move forward on energy literacy using such a system. Users will have a usable system, economic, simple and direct that informs in real time of their habits of consumption, in such a way that they can correct them.

\section{Acknowledgement}

This work has been sponsored by the Spanish Ministry of Economy and Competitiveness through PERSEIDES (ref. TIN2017-86885-R) and CHIST-ERA (ref. PCIN-2016010) projects and by MINECO grant BES-2015-071956. Ramallo-González thanks the program Saavedra Fajardo (grant number220035/SF/16) funded by Consejería de Educación y Universidades of CARM,Fundación SénecaAgencia de Ciencia y Tecnología de la Región de Murcia.

\section{References}

Bangor, A. (2009). Determining What Individual SUS Scores Mean: Adding an Adjective Rating Scale. [online] Semanticscholar.org. hnps://www.semanticscholar.org/paper/DeterminingWhat-Individual-SUS-Scores-Mean-A-an-Bangor.

Barnum, C. (2011). Usability testing essentials. Burlington, MA: Morgan Kaufmann Publishers.

European Building Performance Directive (recast 2011). European Commission.

Fiesta-audit.eu. (2018). Métodos y objetivos. [online] http:// www.gesta-audit.eu/es/el-proyecto/ [18 August 2018].
Gardner, G.T., Stern, P.C. (2002) Environmental problems and human behavior (2nd ed.), Pearson Custom Publishing, Boston, MA

Jervey, B. (2012). Why Energy Literacy Maners \& What to Do About It. [online] TreeHugger. Disponible en: hnps://www.treehugger.com/energy-policy/ whyenergy-literacy-maners-what-to-do-about-it.html.

Pahl, S., Goodhew, J., Boomsma, C., Sheppard, S. (2016). The Role of Energy.

Pellicia, L, Klimant, P., Schumann, M., Pürzel, F., Winstock, V., Putz, M. (2015). Energy visualization techniques for machine tools in virtual reality.

Pérez-Lombard, L., Ortiz, J., Pout, C. (2008). A review on buildings energy consumption information, Energy and Buildings, 40(3),2008,394-398.

Ramallo-González, A.P. et al. Deliverable 5.3: Performance Evaluation and Lessons Learnt, Project ENTROPY . http://entropy-project.eu/wpcontent/uploads/2018/12/D5.3.pdf.

Rashed-Ali, H., Quarles, J., Fies, C. and Sanciuc, L. (2014). Use of augmentedreality in teaching energy efficiency: prototype development and testing.

Sauro, J. (2011). Measuring U: Measuring Usability with the System Usability Scale (SUS). [online] Measuringu.com. Disponible en: hnps://measuringu.com/sus/ [15 de August 2018].

Usability.gov (2013). System Usability Scale (SUS) | Usability.gov. [online] Usability.gov. hnps://www.usability.gov/how-to-and-tools/ methods/system-usability-scale.html 